\title{
QCD in Nuclear Processes at Jefferson Lab
}

\author{
G.P.Gilfoyle \\ University of Richmond - Dept of Physics \\ 28 Westhampton Way, Richmond, VA - USA
}

\begin{abstract}
The scientific program at Jefferson Lab to probe the properties of QCD in the nuclear environment is outlined. The experimental facilities are described and recent results are presented including measurements of color transparency and medium modifications of vector mesons. The evidence for the onset of color transparency is modest and changes to the mass and width of the $\rho$ meson in heavy nuclei are consistent with zero. Finally, the future prospects for the upgraded laboratory are discussed.
\end{abstract}

\section{Introduction}

Quantum chromodynamics (QCD) is our best theory for describing the forces among the fundamental constituents of matter; the quarks and gluons of the Standard Model. Despite many successes, a full, QCD-based understanding of how nucleons and atomic nuclei emerge from these basic building blocks remains elusive. The primary mission of the Thomas Jefferson National Accelerator Facility (Jefferson Lab or JLab) in Newport News, VA, USA is to build a picture of nucleon and nuclear structure in terms of the underlying quarks and gluons bound by the forces of QCD. Here we describe the facilities at Jefferson Lab and recent results relevant to our understanding of QCD in nuclei.

\section{Experimental Facilities}

The main scientific instrument at JLab is the Continuous Electron Beam Accelerator Facility (CEBAF) which can produce electrons of energy up to $6 \mathrm{GeV}$ by recirculating the beam five times through two, superconducting linacs. The maximum current is $200 \mu \mathrm{A}$ with an energy precision of $10^{-4}$, a polarization of $\approx 85 \%$, and a duty cycle of $100 \%$. The beam can by simultaneously injected into three end stations: Halls A, B, and C. The base equipment in Hall A consists of two High Resolution Spectrometers (HRS) with $\Delta p / p<2 \times 10^{-4}$ and a third, large-acceptance spectrometer (BigBite). Experiments here require high luminosity $(\approx$ $10^{38} \mathrm{~cm}^{-2} \mathrm{~s}^{-1}$ ) and high momentum or angular resolution. The CEBAF Large Acceptance Spectrometer (CLAS) is located in Hall B and has an acceptance covering most of $4 \pi$. Its unique toroidal, superconducting magnet is divided into six identical sectors. Each sector consists of drift chambers to measure the charged particle trajectories, a Cerenkov counter to separate electrons and $\pi^{-}$'s, scintillators to measure time-of-flight, and an electromagnetic calorimeter. The resolution of CLAS is $\Delta p / p=0.5 \%$ at forward angles and it can operate at a luminosity of $10^{34} \mathrm{~cm}^{-2} \mathrm{~s}^{-1}$. Experiments with CLAS require moderate resolution, but wide kinematic coverage and multiple-particle final states. A photon tagger upstream from CLAS provides photons of known energy. The base equipment in Hall C consists of the HighMomentum Spectrometer (HMS) with a maximum particle momentum of $7.6 \mathrm{GeV} / \mathrm{c}$ and the Short-Orbit Spectrometer (SOS). The resolution of the HMS is intermediate between Halls $\mathrm{A}$ and $\mathrm{B}\left(\Delta p / p \approx 10^{-3}\right)$ and the SOS has a large momentum acceptance and a short flight path for detecting particles with short lifetimes. Hall $\mathrm{C}$ can operate at high luminosity and is also used for a variety of single-experiment setups.

DIS 2008 


\section{Color Transparency}

One of the long-standing (and surprising) predictions of QCD is color transparency (CT) [1]. At high momentum transfers where the wavelength of the virtual photon is small, a color-neutral, point-like configuration (PLC) may be formed. The small size of the PLC means the color field nearly vanishes and so does the interaction with the surrounding nuclear medium. The PLC can move freely through the now-transparent nucleus with little attenuation. There is no comparable effect expected in the hadronic model so observation of CT would be compelling evidence of quark-gluon effects in nuclei.

Considerable effort has gone into finding evidence of CT. Measurements of $\rho^{0}$ electroproduction at HERMES provide some of the best current evidence for $\mathrm{CT}$ and lie in the range $\mathrm{Q}^{2}=1-2(\mathrm{GeV} / \mathrm{c})^{2}[2]$. The effect is observed by measuring the transparency defined as the ratio of the cross section per nucleon on a nuclear target relative to the same process on a free nucleon. The expectation is that the transparency $T$ will rise with increasing $\mathrm{Q}^{2}$ or mass number $A$.

Experiments at JLab have produced mixed signals. A measurement of the transparency in the quasielastic $A\left(e, e^{\prime} p\right)$ reaction was performed in Hall A for $\mathrm{Q}^{2}<$ $8.1(\mathrm{GeV} / \mathrm{c})^{2}$ using four nuclear targets $(\mathrm{D}, \mathrm{C}$, and $\mathrm{Fe})$ [3]. The transparency was extracted by comparing the data with a calculation in the plane-wave impulse approximation and no evidence of $\mathrm{CT}$ was found in the $\mathrm{Q}^{2}$ or $A$ dependence. A more inviting way to search for color transparency is to look at the electroproduction of mesons because they are smaller than baryons. A recent measure-

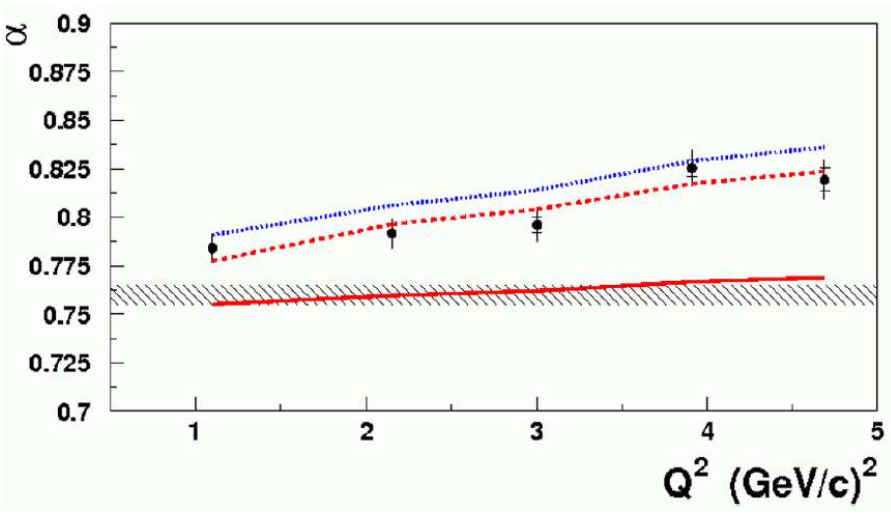

Figure 1: The $\mathrm{Q}^{2}$ dependence of the parameter $\alpha$ for $A\left(e, e^{\prime} \pi^{+}\right)$reaction. The inner error bars are the statistical uncertainties and the outer ones are the sum of statistical, systematic, and model uncertainties in quadrature. Curves are discussed in the text. ment of the $A\left(e, e^{\prime} \pi^{+}\right)$reac-

tion was performed in Hall $\mathrm{C}$ in the range $\mathrm{Q}^{2}=1.1-4.7(\mathrm{GeV} / \mathrm{c})^{2}$ on ${ }^{2} \mathrm{H},{ }^{12} \mathrm{C},{ }^{27} \mathrm{Al},{ }^{63} \mathrm{Cu}$, and ${ }^{197} \mathrm{Au}$ targets [4]. The transparency was extracted as the super-ratio of $\sigma_{A} / \sigma_{H}$ from the data to a model of pion electroproduction from nuclei with $\pi^{+}-N$ final-state interactions. The transparency $T$ at each value of $\mathrm{Q}^{2}$ was then fit to the form $T=A^{\alpha-1}$. The parameter $\alpha$ is $\approx 0.86$ in pion-nucleus scattering and is expected to be independent of energy. The results are shown in Figure 1. The hatched region is the result of pion-nucleus scattering. The solid line is the result of fitting the $\alpha$ dependence of a traditional Glauber calculation without CT. The dashed line is a similar calculation using Glauber and CT and the dotted line is from a calculation including Glauber, CT, and short-range correlations. The data are most consistent with the dashed curve and agree with the previous Jefferson Lab results showing a gradual onset of color transparency and excluding models that predict a rapid

DIS 2008 
transition [5]. Additional measurements of incoherent $\rho$ electroproduction on nuclear targets (e.g. Jefferson Lab Experiment E02-110) are currently being analyzed.

\section{Medium Modifications of Hadronic Properties}

Twenty years ago the observation of the EMC effect revealed that strongly interacting matter described by QCD is different if immersed in a nucleus. Since that first observation a wide variety of approaches have to been taken to study the effect, but the interpretation of the data is often difficult. Observations of vector meson production on fixed targets are a promising avenue. Calculations by Hatsuda et al. [6] are based on QCD sum rule calculations and obtain the following relationship between the free and in-medium masses

$$
\frac{m_{V M}(\rho)}{m_{V M}(\rho=0)}=1-\alpha \frac{\rho}{\rho_{0}}, \quad \alpha=0.16 \pm 0.06,
$$

where $\rho$ is the nuclear density and $\rho_{0}=0.16 \mathrm{fm}^{-3}$. A measurement by the KEK-PS Collaboration of $e^{+} e^{-}$pairs from the decay of vector mesons produced in $12-\mathrm{GeV}$ protons collisions with nuclear targets found $\alpha=0.092 \pm 0.002$ [7]. The CBELSA-TAPS Collaboration has reported $\alpha=0.14$ for the mass of the $\omega$ from the $\pi^{0} \gamma$ decay of low-momentum $\omega$ mesons produced with a photon beam on nuclear targets [8].

An experiment performed with the CLAS detector in Hall B at Jefferson Lab has revealed conflicting results [9]. Tagged photons from a primary electron beam in the range $\mathrm{E}=3-4 \mathrm{GeV}$ were incident on nuclear targets $(\mathrm{D}, \mathrm{C}, \mathrm{Fe}$, and $\mathrm{Ti})$. Vector mesons $(\rho, \omega$, and $\phi)$ were detected in CLAS via their $e^{+} e^{-}$ decay which is less vulnerable to distortions from finalstate interactions (FSI). The large $\pi^{+} \pi^{-}$background was reduced to $10^{-7}$ for two-track events using kinematic cuts. Figure 2 shows the invariant

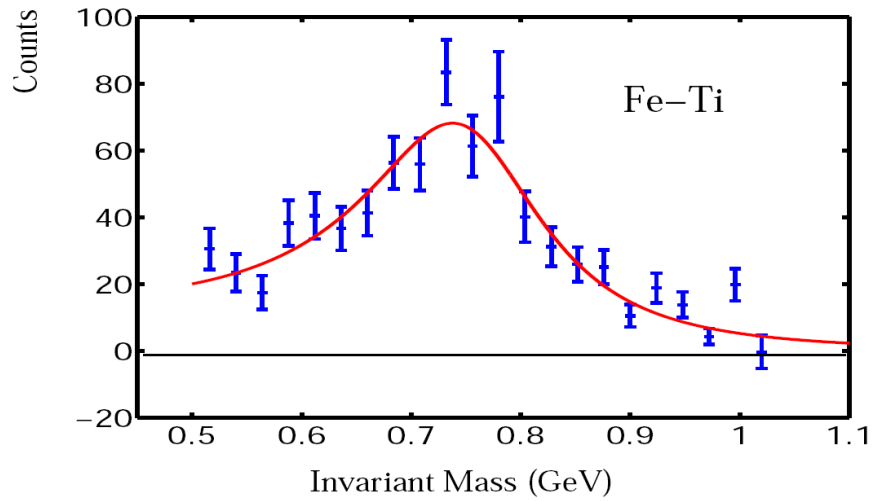

Figure 2: Invariant mass spectra for $e^{+} e^{-}$decay of the $\rho$ photoproduced from an iron-titanium target. The curve is a fit to the spectral shape using the BUU model [10].

$e^{+} e^{-}$mass spectrum for $\rho$ production from the $\mathrm{Fe}-\mathrm{Ti}$ target. The events from the iron and titanium targets were combined in the analysis for better statistics. The $\rho$ should show the greatest effect of the nuclear medium since it has the shortest lifetime and is most likely to decay in the nucleus $(c \tau=1.4 \mathrm{fm})$. The centroid in Figure 2 is little changed from the free hadron value of $770 \mathrm{MeV} / \mathrm{c}^{2}$. Calculations of the mass and width of the $\rho$ were performed using the BUU transport model [10] to account for various nuclear effects (Fermi motion, collisional broadening, etc.), but do not include medium modifications. For all targets there was excellent agreement between the measured mass and width and the BUU calculations. For example, 
the measured $\rho$ mass from the Fe-Ti target was $779.0 \pm 5.7 \mathrm{MeV} / \mathrm{c}^{2}$ versus $773.8 \pm 5.4$ for the BUU model. The value of $\alpha$ extracted from the CLAS measurement is $\alpha=0.02 \pm 0.02$; consistent with no medium effect.

\section{Additional Programs and Future Prospects}

The scientific program at Jefferson Lab is a rich, testing ground for quantum chromodynamics in the nuclear environment. In addition to the work described above there is a program in Hall B to study how deconfined quarks propagate through nuclear matter and build their full color field (hadronize) to form the final state mesons and baryons [11]. This work is not only a unique way to test QCD, but the results are also needed to understand jet production in high-energy, heavy-ion collisions at RHIC [12]. That program is near the end of their analysis. To understand the emergence of nuclei from QCD, short-range correlations are measured in Halls A and B and provide a unique tool to study the nucleon force under high density and at low temperature $[13,14,15]$. These conditions are also relevant to the structure of neutron stars. Finally, an active program to study hypernuclei in Halls A and C will open a new window on how quarks interact in the nuclear environment [16]. Imbedding a strangeness-carrying $\Lambda$ deep inside the nucleus via the $\left(e, e^{\prime} K^{+}\right)$reaction is a novel way to study nuclear structure and dense, cold nuclear matter. Much has been learned and more is on the way.

The future is bright for the study of QCD in the nuclear environment. The accelerator at Jefferson Lab will be doubled in energy to $12 \mathrm{GeV}$, Halls B and $\mathrm{C}$ will be upgraded, and an entirely new end station, Hall D, will be built to take advantage of the new physics opportunities. The $12-\mathrm{GeV}$ Upgrade is the highest rated priority in nuclear physics in the United States. The scientific program after the Upgrade is already slated to include investigations of hadronization in nuclei (Jefferson Lab Experiments E12-06-117, E12-06-101), color transparency (Experiments E12-06-106 and E12-06-107), and short-range correlations (Experiment E12-06-105) in the first five years of running after the Upgrade.

\section{References}

[1] W. Kittel et al., editors. World Scientific, Singapore, 1983.

[2] A. Airapetian et al. Phys. Rev. Lett., 90(5):052501, Feb 2003.

[3] K. Garrow et al. Phys. Rev. Lett., 66:044613, 2002.

[4] B. Clasie et al. Phys. Rev. Lett., 99:242502, 2007.

[5] J. Dutta et al. Phys. Rev. C, 68:021001, 2003.

[6] T. Hasuda et al. Phys. Rev. C, 46:R34, 1992.

[7] R. Muto et al. Phys. Rev. Lett., 98:042501, 2007.

[8] M. Naruki et al. Phys. Rev. Lett., 96:092301, 2006.

[9] R. Nasseripour et al. Phys. Rev. Lett., 99:262302, 2007.

[10] P. Muehlich et al. Phys. Rev. C, 67:024605, 2003.

[11] W. Brooks. Quark propagation through cold qcd matter. Proposal e02-104, Jefferson Lab, Newport News, VA, 2002.

[12] B. Z. Kopeliovich, J. Nemchik, and Ivan Schmidt. Nucl. Phys., A782:224-233, 2007.

[13] K. S. Egiyan et al. Phys. Rev. Lett., 96:082501, 2006.

[14] R. Shneor et al. Phys. Rev. Lett., 99:072501, 2007.

[15] R. Subedi et al. Science, 320:1476, 2008.

[16] M. Iodice et al. Phys. Rev. Lett., 99:052501, 2007. 